\title{
Cytopathological Study of Head \& Neck Swellings in Tertiary Care Centre
}

\author{
Dr. Bollipogu Srilatha ${ }^{1}$, Dr. Shyamala Srujana ${ }^{2 *}$, Dr. SSS Quadri ${ }^{3}$, Dr. Naval Kishore Bajaj ${ }^{4}$
}

${ }^{1}$ Assistant Professor, Government Medical College, Mahabubnagar Metugadda, Mahabubnagar District, Telangana 509001, India
${ }^{2}$ Associate professor, Government Medical College, Mahabubnagar Metugadda, Mahabubnagar District, Telangana 509001, India
${ }^{3}$ Associate professor, Government Medical College, Mahabubnagar Metugadda, Mahabubnagar District, Telangana 509001, India
${ }^{4}$ Professor \& HOD, Government Medical College, Mahabubnagar, Mahabubnagar Metugadda, Mahabubnagar District, Telangana 509001, India

DOI: 1 10.36348/SJPM.2019.v04i12.002 $\quad$ | Received: 02.12.2019| Accepted: 09.12.2019| Published: 11.12 .2019

*Corresponding author: Dr. Shyamala Srujana

Abstract

Introduction: Fine needle aspiration cytology is rapid, virtually non invasive and simple diagnostic procedure .FNAC offers a simple method of diagnosis of neoplastic and non neoplastic lesions of the head and neck. Material and methods: 280 cases of cervical lesions were taken up in the study over a period for 2 years from August 2017 to July 2019. Various staining procedure was done based on the sample. Results: Peak incidence of 90 (32.1\%) cases was noted in 21-30 years age group. There were $168(60 \%)$ females among 280. Site wise distribution shows that majority of lesions are from lymph nodes (39.2\%). Of which Granulomatous lymphadenitis of Tuberculous etiology of about 60 cases. Discussion and conclusion: FNAC is a procedure which can be carried out as an out-patient procedure with minimal problems. Owing to its accurate diagnostic potential it is proved useful in separating inflammatory, benign and malignant lesions with good certainty.

Keywords: FNAC, cervical lesions, tuberculosis, Lymph Node, Haemotoxylin and Eosin stain.

Copyright @ 2019: This is an open-access article distributed under the terms of the Creative Commons Attribution license which permits unrestricted use, distribution, and reproduction in any medium for non-commercial use (NonCommercial, or CC-BY-NC) provided the original author and sources are credited.

\section{INTRODUCTION}

Fine needle aspiration cytology is a well known safe diagnostic procedure. It is a cost-effective and first-line investigation. Fine needle aspiration cytology is rapid, virtually non invasive and simple diagnostic procedure, the importance and applicability of which is being increasingly appreciated by clinicians and pathologists worldwide in recent time. FNAC offers a simple method of diagnosis of neoplastic and non - neoplastic lesions of the head and neck. The procedure is safe and free from complications and is well tolerated by the patients. This technique has been successfully utilized in the diagnosis of many pathological lesions in multiple organs including lung, lymph nodes, bone, thyroid, salivary gland, soft tissue as well as other anatomic regions like head and neck, thorax, abdomen and pelvis.

FNAC does not give the same architectural detail as histology but it can provide cells from the entire lesion as many passes through the lesion can be made while aspirating. The present study was undertaken to determine the role of FNAC in the evaluation of cytomorphological features of various Head \& Neck lesions.

\section{MATERIALS AND METHODS}

A present study of 280 cases was done in the Department of Pathology for 2 years from August 2017 to July 2019. One year of Retrospective and one year of Prospective. Those patients who presented with superficially palpable head and neck lesion in Medicine, Surgical, Dermatology are adviced for FNAC procedure. After taking present and past history of the present swelling and after local examination, the palpable swelling was fixed with one hand and with all aseptic precautions, $22-23 \mathrm{G}$ needle with $10 \mathrm{ml}$ syringe was inserted into the swelling and a negative pressure was applied. The needle was moved back and forth as well as in different directions within the mass so as to collect sample from different areas around. Throughout the procedure negative pressure was maintained in the syringe by keeping the plunger retracted. In case blood was aspirated, the procedure was stopped and the needle was withdrawn only to repeat the same at different site. When the aspiration was felt to be complete, the plunger was released to equalise the pressure in the syringe before withdrawing the needle, thereby preventing the loss of cells from the lumen of the needle to syringe. The syringe was then detached from the needle and the latter was then withdrawn. Pressure with sterile gauze piece was applied at the site 
of aspiration for some time, to stop any leakage of blood.

The aspiration material was smeared on the glass slides, by taking two $\mathrm{ml}$ of air in to the syringe and was reattached to the needle. Keeping the tip of the needle on the surface of a clean, grease free glass slide, the plunger of the syringe was advanced so as to force the aspirated material in the needle on to the slide and smears are made on the slide with help of spreader slide, later slides were fixed in alcohol and stained relevantly with stains, including May Grunwald Giemsa, Papanicolaou and Haemotoxylin and Eosin (H\&E) stains and Papanicolaou stain. Air-dried, smears was stained with Leishman stain, Toulidine blue stain and the others are kept unstained to be used for ZiehlNeelsen (ZN) staining whenever a cytological diagnosis of Granulomatous disease was made and also in cases with abundant necrosis and suppuration. In cases where fluid was aspirated, the fluid was centrifuged and smears were made from the sediment, followed by the above staining methods.

Cytological findings were recorded and patients were advised medical treatment and follow up or biopsy and surgical intervention depending upon the pathology.

\section{RESULTS}

There were $168(60 \%)$ females and 112(40\%) were males (Chart-1). The age-wise distribution of the patients in this study is shown in Table-1.

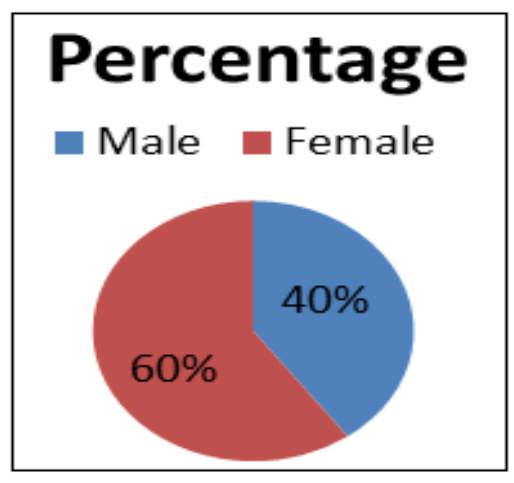

Chart-1: Sex distribution
The age range of the 280 patients in this study was between 2 months to 80 years. Peak incidence of $90(32.1 \%)$ cases was noted in 21-30 years age group, followed by $75(26.7 \%)$ was recorded in age group of 31-40 years of life. Least incidence was noted in age group of 71-80 yrs of 2(0.7\%).

Table-1: Age-wise distribution of the patients

\begin{tabular}{|l|l|l|}
\hline Age in Years & No. of Patients & Percentage \\
\hline $0-10$ & 10 & 3.57 \\
\hline $11-20$ & 40 & 14.28 \\
\hline $21-30$ & 90 & 32.1 \\
\hline $31-40$ & 75 & 26.7 \\
\hline $41-50$ & 33 & 11.7 \\
\hline $51-60$ & 20 & 7.1 \\
\hline $61-70$ & 10 & 3.5 \\
\hline $71-80$ & 02 & 0.7 \\
\hline
\end{tabular}

Table-2, showing Site wise distribution of FNAC of head and neck lesions of present study which shows that majority of lesions are from lymph nodes, 110(39.2\%), followed by Skin and soft tissue lesions 75 cases of $26.7 \%$. Thyroid lesions were $60(21.4 \%)$, followed by salivary gland lesions $30(10.7 \%)$ cases with least number of cases sampled from oral cavity, i.e. $5(1.7 \%)$ cases. Of the total 280 cases with head and neck lesions, $262(93.5 \%)$ cases were non-neoplastic in nature, $18(6.4 \%)$ cases were malignant.

Table-2: Site wise distribution of FNAC of head and neck lesions

\begin{tabular}{|l|l|}
\hline Site of FNAC & No. $(\%)$ \\
\hline Lymph Node & $110(39.2 \%)$ \\
\hline Thyroid & $60(21.4 \%)$ \\
\hline Salivary gland & $30(10.7 \%)$ \\
\hline Oral cavity & $05(1.7 \%)$ \\
\hline Skin and soft tissue lesions & $75(26.7 \%)$ \\
\hline TOTAL & 280 \\
\hline
\end{tabular}


Table-3: Showing Distribution of Head \& Neck Lesions according to FNAC diagnosis.

\begin{tabular}{|l|l|l|l|}
\hline Benign Lesions & No. $(\%)$ & Malignant Lesions & No. $(\%)$ \\
\hline LYMPH NODE & & & \\
\hline Reactive hyperplasia (Fig-1) & $25(8.9)$ & Lymphoma & $02(0.7)$ \\
\hline Granulomatous lymphadenitis (Fig-2) & $60(21.4)$ & Metastatic deposits in lymph node & $08(2.8)$ \\
\hline Acute Suppurative & $10(3.5)$ & & \\
\hline Chronic Necrotizing lesions & $05(1.78)$ & & \\
\hline THYROID & & & \\
\hline Hashimoto's Thyroiditis (Fig-3) & $30(10.7)$ & Papillary carcinoma & $03(1.0)$ \\
\hline Colloid goiter & $25(8.9)$ & & \\
\hline Hyperplasia of Thyroid & $02(0.7)$ & & \\
\hline SALIVARY GLAND & & & \\
\hline Sialadenitis & $20(7.1)$ & & \\
\hline Pleomorphic Adenoma & $10(3.5)$ & & $03(1.0)$ \\
\hline SKIN AND SUBCUTNEOUS LESIONS & & & $02(0.7)$ \\
\hline Benign lipomas & $30(10.7)$ & Squamous cell carcinoma & \\
\hline Keratinous Cysts & $20(7.1)$ & Basal cell carcinoma & \\
\hline Neuro fibroma & $10(3.5)$ & & \\
\hline Benign skin Tumors & $02(0.7)$ & & \\
\hline ORAL CAVITY & & & \\
\hline Abcess & $04(1.4)$ & & \\
\hline Retension cyst & $01(0.3)$ & & \\
\hline
\end{tabular}

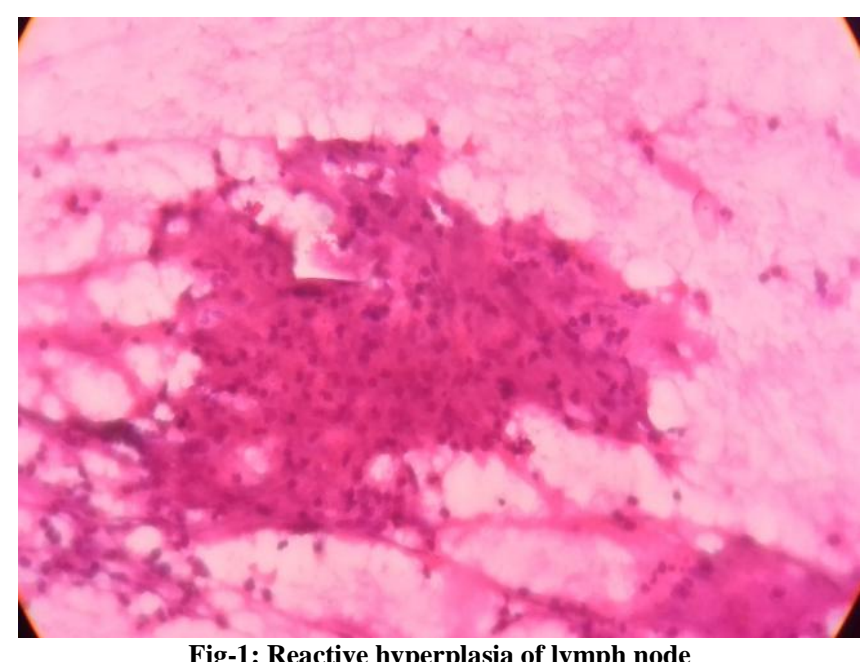

Fig-1: Reactive hyperplasia of lymph node
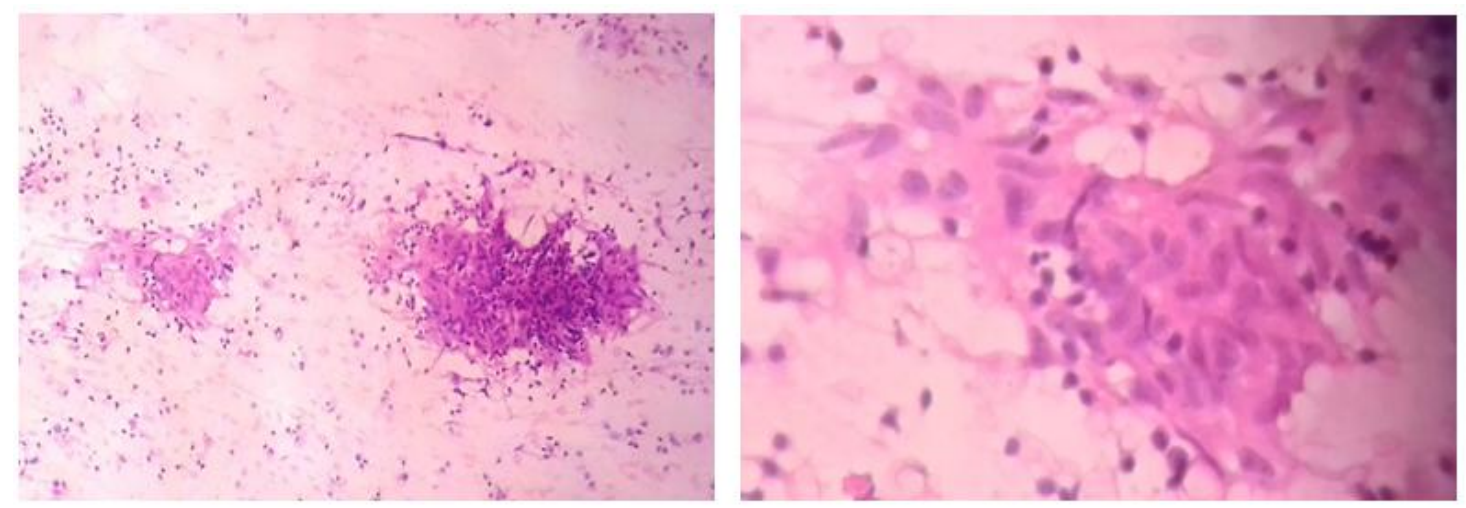

Fig-2: Granulomatous lymphadenitis with epithelioid cell clusters 

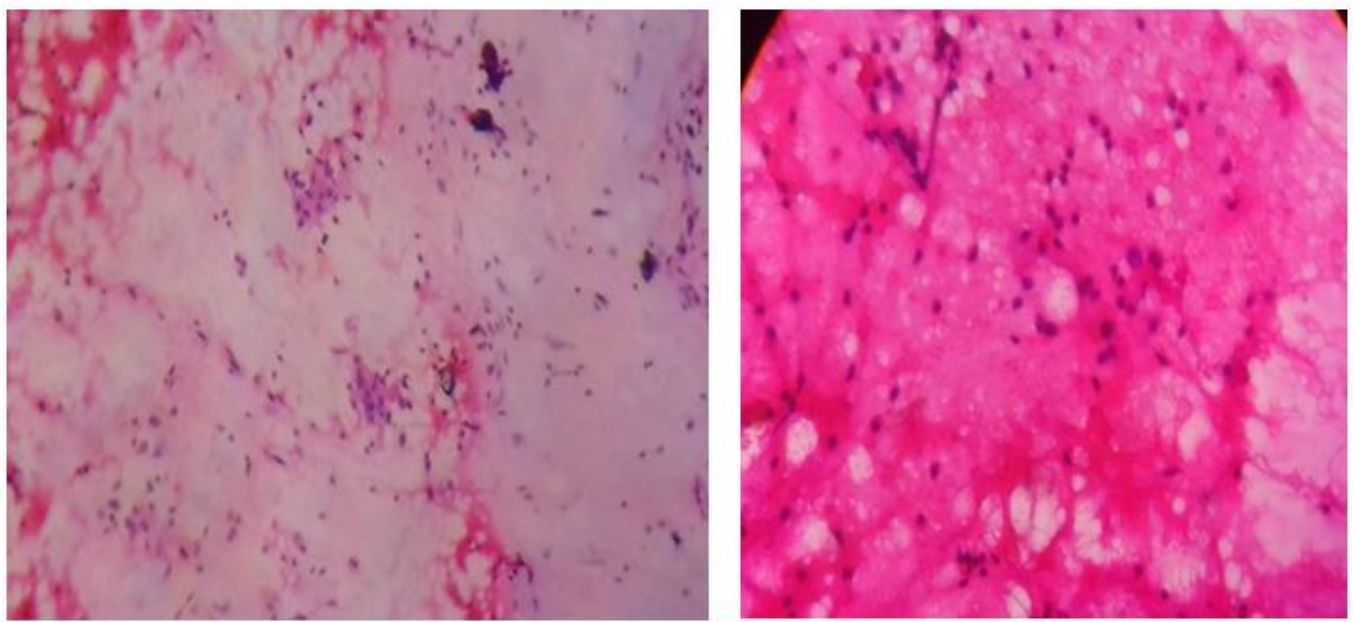

Fig-3: Hashimoto's Thyroiditis

We encountered majority of cases of Granulomatous lymphadenitis of Tuberculous etiology of about 60 cases, which shows the disease load in our region. Of which we encountered 4 Cytological patterns of Epithelioid granulomas with and without caseous necrosis, Acute suppuration with necrosis and Necrosis only with chronic inflammatory cells and no granulomas, detailed case wise dstridution is shown in Table-4. Of which AFB staining was positive in 12 cases and remaining 48 cases were AFB negative.

Table-4: Cytomorphological Pattern of Tuberculosis Lymphadenitis in our study

\begin{tabular}{|l|l|}
\hline Cytological Picture & No. of Cases \\
\hline Epithelioid granulomas with Necrosis & 26 \\
\hline Epithelioid granulomas without Necrosis & 20 \\
\hline Acute suppuration with necrosis & 10 \\
\hline Necrosis only with chronic inflammatory cells and no granulomas & 04 \\
\hline
\end{tabular}

\section{DISCUSSION}

Diagnostic cytology when performed by well trained experienced individual offers high degree of reliability and feasibility, therefore FNAC has gained universal acceptance as in most instances it is inexpensive, safe, quick and accurate. Head and Neck masses often pose a challenging diagnostic problem to the clinician. The goal of FNAC in the head and neck area as in other anatomical sites is to provide clinically useful information which exceeds that obtained by palpation or imaging alone. The false negatives and false positives were pointers towards problems and pitfalls in cytological interpretation [19].

FNAC is defined as using a fine needle to remove sample of cells from suspicious masses for diagnostic purposes. Fine needle aspiration cytology has revolutionized the diagnosis of lymphadenopathy, decreasing the morbidity of excisional or incisional biopsy of lymph node [7, 8] especially in developing countries like INDIA with limited financial \& health care resources. Majority of enlarged lymph nodes represent benign, reactive or inflammatory process.

S. Soni et al., [17] Studied 59 cases and found $47.45 \%$ were of neck nodes, $23.72 \%$ were of thyroid, $22.03 \%$ were of salivary glands and $6.77 \%$ were of other lesions. Farzana Shahid et al., [18] have studied 518 FNAC from head and neck lesions, out of which
473 were benign and 45 cases were malignant. Maximum number of cases were from Lymphnodes (356), followed by Thyroid (124) and Salivary gland (38). The above 2 studies are almost similar to our analysis. In Lakshmi Bai et al., [16] study 43(9.83\%) cases were malignant and 394 cases (90.16\%) were benign. The frequency of the various lesions in the different organs were as follows Lymph-Nodes 199cases (45.5\%), Thyroid-141cases (32.5\%), Salivary Gland-28 cases $(6.4 \%)$ and others 69 cases $(15.9 \%)$.

The above studies are almost similar to our analysis. Majority of lesions are from lymph nodes, $110(39.2 \%)$, followed by Skin and soft tissue lesions 75 cases of $26.7 \%$.Thyroid lesions were $60(21.4 \%)$, followed by salivary gland lesions 30(10.7\%) cases with least number of cases sampled from oral cavity, i.e. $5(1.7 \%)$ cases. Of the total 280 cases with head and neck lesions, $262(93.5 \%)$ cases were non-neoplastic in nature, $18(6.4 \%)$ cases were malignant.

A major proportion of lymphadenopathies in this study were also due to benign conditions of 60 $(21.4 \%)$ cases. This is in accordance with the studies by Shrivastava et al., and Ahmad et al., in which lesions were benign $88 \%$ and $86.4 \%$ respectively $[8,9]$. Female preponderance which is seen in the present study was similar to Sanjay Chauhan et al., and Williams et al., studies $[10,11]$. But male preponderance was noted in 
other studies like Patar Mukul et al., and Anne et al., $[12,13]$.

An important cause of superficial lymphadenopathy in India is tuberculosis. Various types of cytological appearances have been described in tubercular lymphadenitis which include the following four categories: epithelioid granulomas with caseous necrosis, epithelioid granulomas without necrosis, necrosis only without epithelioid granulomas and polymorphs with necrosis with or without epithelioid granulomas $[4,5]$.

The combination of FNAC with $\mathrm{ZN}$ staining for AFB is of utmost significance as a diagnostic modality in these lesions. We were able to demonstrate the presence of acid fast bacilli on Zeihl Neelsen stain in 12 cases. 26 cases showed classical epithelioid granulomas with caseous necrosis, 4 cases were reported as chronic necrotizing lymphadenitis, 10 cases showed acute suppuration with presence of neutrophils and 20 cases showed epithelioid granulomas without caseation. Thus, $21.4 \%$ of all enlarged lymph nodes in this study had a Koch's etiology. The predominance of cervical nodal involvement in tuberculosis has been established by many studies $[8,14,15]$, which could be attributed to infection of the tonsils and adenoids providing portal of entry. Tuberculous lymphadenopathy is the commonest manifestation of extra-pulmonary tuberculosis where cervical groups of lymph nodes are most frequently involved. In the present study 02 cases of Hodgkins lymphoma and 8 cases of Metastatic deposits to lymph nodes are seen.

FNAC reduces the rate of unnecessary thyroid surgery for patients with benign nodules and appropriately triages patients with malignancy to the required surgery. FNAC procedure serve a therapeutic function since the aspiration of fluid in cysts may be followed by involution of the lesion [6].

Farzana Shahid et al., [18] where in their commonest lesion was Benign Nodular Goitre (52.4\%); Inflammatory lesions (7.4\%) and Neoplastic were 3.2\%. Another study conducted by Lakshmibai B et al., [16] with regards to thyroid lesions showed that Nodular Goitre $(41.9 \%)$ was the commonest followed by Inflammatory lesions such as Hashimotos's Thyroiditis $37.6 \%$, Hyperplastic Thyroid-2.8\%, Lymphocytic Thyroiditis $1.4 \%$. The neoplastic lesions constituted about $6.4 \% .3$ cases of normal thyroid and in 10 cases $(7 \%)$ the aspirates were inconclusive.

In present study Hashimoto's Thyroiditis constitute about $30(10.7 \%)$ cases, colloid goiter of 25 $(8.9 \%)$ cases and Hyperplastic thyroid of02 (0.7\%) cases. We encountered $03(1.0 \%)$ cases of papillary carcinoma.
With regards to salivary glands in our study there were 30 cases $(10.7 \%)$. The commonest lesion being inflammatory-Chronic Sialadenitis 8 cases $(7.1 \%)$, 10cases $(3.5 \%)$ benign neoplasms- Pleomorphic Adenoma and we did not encounter any malignant salivary gland neoplasm.

Study by El Hag et al., [20] also showed that inflammatory lesions such as sialadenitis was most commonest with 12 cases $(57 \%)$ followed by benign lesions 8 cases (38\%) and mucoepidermoid carcinoma 1 case $(5 \%)$ which is similar to our study.

A study conducted by Maniyar et al., [21] showed 10 benign and 7 malignant cases of soft tissue lesions, but in our study Benign Lipoma cases of 30, Keratinous Cysts were 20 cases and 10 cases of Neurofibroma were encountered.

We studied 02 cases of Benign skin tumors and 03 cases of Squamous cell carcinoma and 02 cases of Squamous cell carcinoma, totally 04 cases of Malignant skin tumors.

Remaining Oral cavity lesions constitute about Oral abcess of 04 cases and Retention cyst of 01 case.

\section{CONCLUSION}

FNAC is a simple and rapid procedure which can be carried out as an out-patient procedure with minimal problems. Owing to its accurate diagnostic potential it is proved useful in separating inflammatory, benign and malignant lesions with good certainty. Moreover, the procedure is simple and cost-effective. Hence, FNAC is recommended as the first-line investigation in diagnosing head and neck lesions.

\section{REFERENCES}

1. Chauhan, S., Patel, M., Bhavsar, M., \& Chavda, B. (2016). Cytopathological Study Of Head \& Neck Swellings In Tertiary Care Centre. Int $J$ Res Med, 5(3), 106-109.

2. Mallappa, L. B., Ramya, B. S., Latha, B., \& Prabhu, D. C. (2011). Profile Of Fine Needle Aspiration Cytology Of Head And Neck Lesions In A Tertiary Hospital. International Journal of Current Research and Review; 3(10):99-105.

3. Svante, R. O. (1995). Manual and Atlas of FNAC 2nd edition.

4. Heerde, P. V., Miliauskas, J., \& Field, A. (2005). Lymphnodes. In: Orell, S. R., Sterrett, G. F., \& Whitaker, D., ed Fine needle aspiration cytology; 4th ed; New York: Churchill Livingstone; 83124.

5. Khetrapal, S., Jetley, S., Jairajpuri, Z., Rana, S., \& Kohli, S. (2015). FNAC of head \& neck lesions and its utility in clinical diagnosis: a study of 290 cases. Thyroid, 49(16.9):33-38.

6. Cibas, E. S., \& Ali, S. Z. (2009). The Bethesda system for reporting thyroid cytopathology. Thyroid, 19(11), 1159-1165. 
7. Qasmi, S. A., Kiani, F., Malik, A. I., Salamatullah, J., Farooq, M. O., \& Abassi, M. A. (2012). Cervical lymphadenopathy: A common diagnostic dilemma. Journal of Surgery Pakistan (International), 17(2), 76-80.

8. Shrivastava, J. P., Shrivastava, A., Singh, S., \& Gaur, R. (2015). Role of FNAC in the evaluation of cervical lymph nodes: a hospital based study. $J$ Evolution Med and Dent Sci, 4(55), 9643-8.

9. Ahmad, S. S., Akhtar, S., Akhtar, K., Naseem, S., \& Mansoor, T. (2005). Study of fine needle aspiration cytology in lymphadenopathy with special reference to acid-fast staining in cases of tuberculosis. JK science, 7(1), 1-4.

10. Chauhan, S., Patel, M., Bhavsar, M., \& Chavda, B. (2016). Cytopathological Study Of Head \& Neck Swellings In Tertiary Care Centre. Int $J$ Res Med, 5(3), 106-109.

11. Betsill JR, W. L., \& Hajdu, S. I. (1980). Percutaneous aspiration biopsy of lymph nodes. American journal of clinical pathology, 73(4), 471-479.

12. Mukul, P., \& Kusum, B. (2014). A clinicopathological evaluation of cervical lymphadenopathy in children (0-14 Years) by fine needle aspiration cytology and histopathological examination-A hospital based study. National Journal of Otorhinolaryngology and Head \& Neck Surgery, 2(11).

13. Wilkinson, A. R., Mahore, S. D., \& Maimoon, S. A. (2012). FNAC in the diagnosis of lymph node malignancies: A simple and sensitive tool. Indian journal of medical and paediatric oncology: official journal of Indian Society of Medical \& Paediatric Oncology, 33(1), 21-24.
14. Sandhaus, L. M. (2000). Fine-needle aspiration cytology in the diagnosis of lymphoma: the next step. American journal of clinical pathology, 113(5), 623-627.

15. Dandapat, M. C., Mishra, B. M., Dash, S. P., \& Kar, P. K. (1990). Peripheral lymph node tuberculosis: a review of 80 cases. British Journal of Surgery, 77(8), 911-912.

16. Mallappa, L. B., Ramya, B. S., Latha, B., \& Prabhu, D. C. (2011). Profile Of Fine Needle Aspiration Cytology Of Head And Neck Lesions In A Tertiary Hospital. International Journal of Current Research and Review; 3(10):99-105.

17. Soni, S., Pippal, S. K., Yashveer, B., Srivastava, P., Soni, S., \& GMC, B. (2010). Efficacy of fine needle aspiration cytology in diagnosis of neck masses. World Articles in Ear, Nose and Throat www. waent. org, 3:1-5.

18. Shahid, F., Mirza, T., Mustafa, S., Sabahat, S., \& Sharafat, S. (2010). An experiential status of fine needle aspiration cytology of head and neck lesions in a tertiary care scenario. Journal of Basic \& Applied Sciences, 6(2):159-162.

19. Fernandes, H., D’souza, C. R. S., \& Thejaswini, B. N. (2009). The role of fine needle aspiration cytology in palpable head and neck masses. Journal of clinical and diagnostic research, 3(5), 1719-1725.

20. Chiedozi, L. C., \& Kollur, S. M. (2003). Fine needle aspiration cytology of head and neck masses. Seven years' experience in a secondary care hospital. Acta cytologica, 47(3), 387-392.

21. Maniyar, A. U., Patel, H. L., \& Parmar, B. H. (2013). Study of Cytodiagnosis of Head and Neck Neoplastic Lesions and comparision with histopathology. RRJMHS, 2(2), 54-9. 